\title{
Features
}

\section{Meeting Report: Outcomes from the Undergraduate Research Summit}

\author{
Thomas J. Wenzel
}

Department of Chemistry, Bates College, Lewiston, ME 04240

There has been some concern expressed in recent times whether science departments at predominantly undergraduate institutions (PUIs) have responded rapidly enough to the changing landscape of higher education and research. These concerns include the increasingly multidisciplinary nature of scientific investigations; the changing demographics of students and faculty members; the increasing desire to integrate research more fully throughout the curriculum; and the increasing pressure to assess whether educational activities, including research, actually improve student learning. These changes present challenges but also opportunities if we take advantage of them in creative ways.

In response to these concerns, the Chemistry division of the National Science Foundation supported the Undergraduate Research Summit (http://abacus.bates.edu/acad/ depts/chemistry/twenzel/summit.html). This summit, which was held in August 2003 at Bates College in Lewiston, ME, brought together an array of stakeholders from the chemistry community, ranging from those with a long history of success in undergraduate research to junior faculty members who have started on a trajectory of success. Participants numbered about 50 and represented a variety of constituencies, including public and private undergraduate institutions (faculty members and administrators), doctoralgranting institutions, industry, national laboratories, and funding agencies. The complete list of participants is available on the summit Web site (http://abacus.bates.edu/ acad/depts/chemistry/twenzel/participant_list.pdf). Participants examined the issues involved in undertaking and sustaining chemistry research at PUIs. Discussion at the meeting was facilitated by a series of white papers that addressed topics such as defining undergraduate research, assessing undergraduate research, discerning the value of diversity within the chemical sciences, designing a researchsupportive curriculum, and addressing the value of collaborations, the importance of an appropriate infrastructure to support research, the value of initiating and sustaining research productivity throughout an academic career, and the potential barriers to undergraduate research at public comprehensive institutions. A report on the outcomes of the summit has been posted online and provides recommendations on how to enhance the number, quality, productivity, and visibility of research programs at PUIs (http://

DOI: $10.1187 /$ cbe.04-05-0043

Email address: twenzel@bates.edu abacus.bates.edu/acad/depts/chemistry/twenzel/ white_papers.html).

Recommendations in the report are aimed at individuals, departments, institutions, funding agencies, and other organizations with an interest in undergraduate research. Summit participants were uniform in their belief that research performed by undergraduates should represent an original contribution to the discipline and that successful projects are intended for publication in peer-reviewed journals. The need for, and value of, activities designed to assess undergraduate research is described in the report. An emphasis of the summit meeting was to explore the utilization of undergraduate research as a means of recruiting and retaining members of historically under-represented groups to chemistry. Recommendations for effective practices to improve diversity within the chemical sciences are described in the report. The importance of having a researchsupportive curriculum and designing strategies for developing such a curriculum were emphasized as well. Another focus was on the value of developing collaborations, partnerships, and alliances as a way of enhancing the research productivity of faculty at PUIs. Ways in which individuals, departments, and institutions can create a culture of undergraduate research are included in the report. An important feature is that many of the recommendations in the report do not require additional resources for their implementation, but instead, require only changes in individual, departmental, or institutional practices.

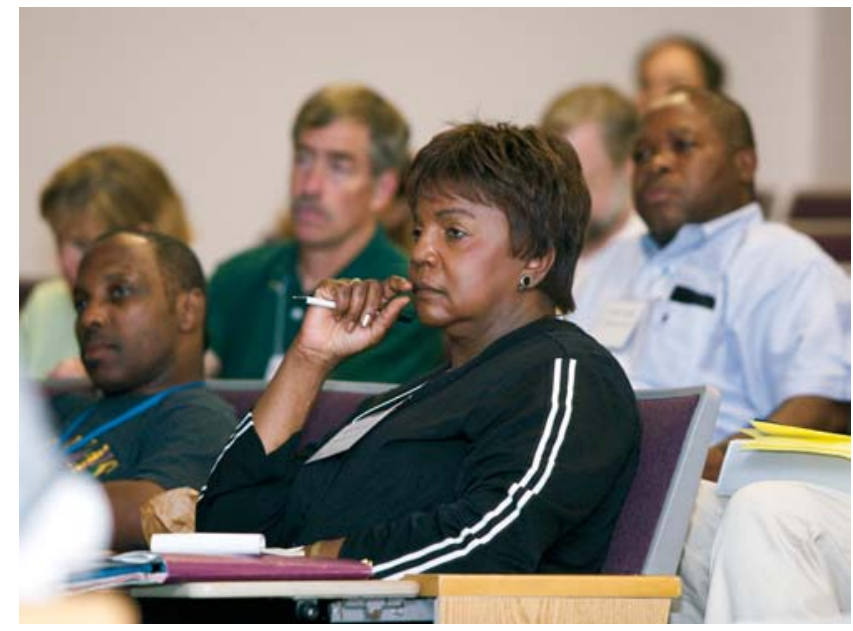




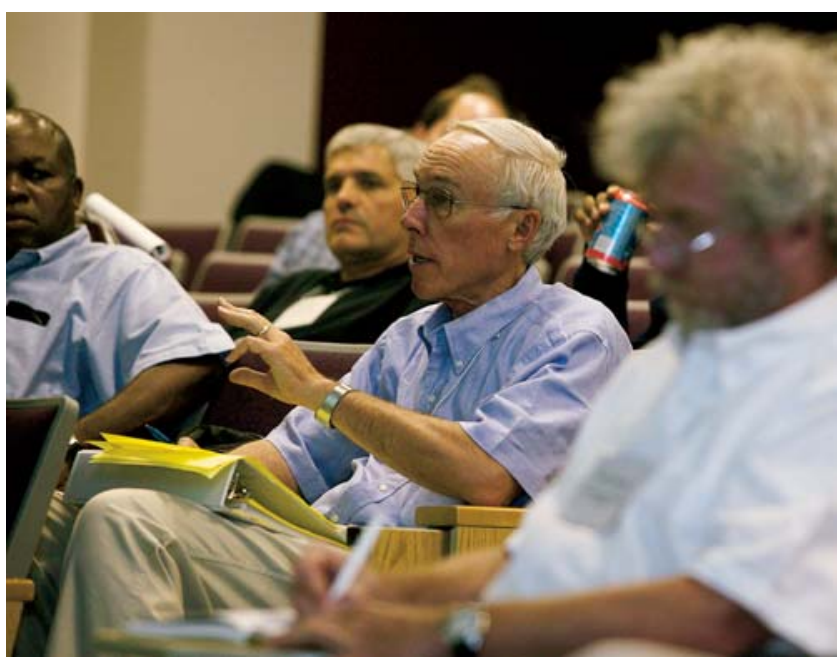

Even though the meeting focused on research in the chemical sciences and was attended almost exclusively by chemists, many of the recommendations contained in the report apply more broadly across all disciplines, especially across science disciplines. The original proposal to the National Science Foundation (NSF) (http://abacus.bates. edu/acad/depts/chemistry/twenzel/summitpropofin.pdf), white papers, final report, and upcoming dissemination activities can all be obtained on the summit Web site at: http://abacus.bates.edu/acad/depts/chemistry/twenzel/ summit.html. Comments on and responses to the report can be sent to Tom Wenzel E-mail: (twenzel@bates.edu). These will be added as appropriate to the summit Web site.

Initial dissemination activities in 2004 have involved a session at the Gordon Research Conference on Chemical Education, a session at the national meeting of the Association of American Colleges and Universities, and symposia and an accompanying workshop at the spring national meeting of the American Chemical Society (http:// abacus.bates.edu/acad/depts/chemistry/twenzel/ dissemination_activities.html). A number of workshops on the outcomes of this summit will be offered at the upcoming national meeting of the Council on Undergraduate Research (http://www.cur.org/conferences/UW-LaCrosse/ program.html). Response to the summit report, as evidenced by the attendance at these sessions and the ensuing discussion among participants, has been especially gratifying. Participants commented about the number of ideas that came out of the sessions that were easily implemented and that they looked forward to taking back to their department and institution. For example, departments have the ability to design a research-supportive curriculum that enhances opportunities for students and faculty to conduct research as part of the major requirements. Departments have the ability to devise teaching schedules that create blocks of time for research. Institutions have the ability to institutionalize programs and activities that send a clear message that undergraduate research is a valued activity.

Additional suggestions for appropriate venues for dissemination of the report and the recommendations therein are welcome. 